\title{
Morphologic and functional changes in the unilateral 6-hydroxydopamine lesion rat model for Parkinson's disease discerned with $\mu$ SPECT and quantitative MRI
}

\author{
Nadja Van Camp - Ruth Vreys - Koen Van Laere - Erwin Lauwers • Dirk Beque • Marleen Verhoye • \\ Cindy Casteels • Alfons Verbruggen - Zeger Debyser • Luc Mortelmans • Jan Sijbers • Johan Nuyts • \\ Veerle Baekelandt • Annemie Van der Linden
}

Received: 22 September 2008 / Revised: 11 January 2010 / Accepted: 18 January 2010 / Published online: 19 February 2010 (C) ESMRMB 2010

\begin{abstract}
Object In the present study, we aimed to evaluate the impact of neurodegeneration of the nigrostriatal tract in a rodent model of Parkinson's disease on the different MR contrasts $\left(\mathrm{T}_{2}, \mathrm{~T}_{1}, \mathrm{CBF}\right.$ and $\left.\mathrm{CBV}\right)$ measured in the striatum.

Material and methods Animals were injected with 6-hydroxydopamine (6OHDA) in the substantia nigra resulting in massive loss of nigrostriatal neurons and hence dopamine depletion in the ipsilateral striatum. Using 7T MRI imaging, we have quantified $\mathrm{T}_{2}, \mathrm{~T}_{1}, \mathrm{CBF}$ and $\mathrm{CBV}$ in the striata of $6 \mathrm{OHDA}$ and control rats. To validate the lesion size, behavioral testing, dopamine transporter $\mu$ SPECT and tyrosine hydroxylase staining were performed.

Results No significant differences were demonstrated in the absolute MRI values between 6OHDA animals and controls;
\end{abstract}

N. Van Camp $(\bowtie) \cdot$ R. Vreys $\cdot$ M. Verhoye $\cdot$ A. Van der Linden Bio-Imaging Lab, University of Antwerp, Groenenborgerlaan 171, 2020 Antwerp, Belgium

e-mail: nadja.vancamp@ua.ac.be

K. Van Laere $\cdot$ D. Beque $\cdot$ C. Casteels $\cdot$ L. Mortelmans $\cdot$ J. Nuyts Division of Nuclear Medicine and MoSAIC, University Hospital Leuven and K.U. Leuven, Leuven, Belgium

E. Lauwers · V. Baekelandt

Laboratory for Neurobiology and Gene Therapy, Leuven, Belgium

M. Verhoye $\cdot$ J. Sijbers

Visielab, University of Antwerp, Antwerp, Belgium

A. Verbruggen

Laboratory for Radiopharmacy, K.U. Leuven, Leuven, Belgium

\section{Z. Debyser}

Laboratory for Molecular Virology and Gene therapy, Division of Molecular Medicine, Department of Molecular and Cellular Medicine, K.U. Leuven, Leuven, Belgium however, 6OHDA animals showed significant striatal asymmetry for all MRI parameters in contrast to controls.

Conclusions These PD-related asymmetry ratios might be the result of counteracting changes in both intact and affected striatum and allowed us to diagnose PD lesions. As lateralization is known to occur also in PD patients and might be expected in transgenic PD models as well, we propose that MR-derived asymmetry ratios in the striatum might be a useful tool for in vivo phenotyping of animal models of PD.

Keywords Parkinson's disease $\cdot$ Rat $\cdot$ Neurodegeneration · SPECT · MRI

$\begin{array}{ll}\text { Abbreviations } \\ \text { 6OHDA } & \text { 6-Hydroxydopamine } \\ \text { CBF } & \text { Cerebral blood flow } \\ \text { CBV } & \text { Cerebral blood volume } \\ \text { DA } & \text { Dopamine } \\ \text { DAT } & \text { Dopamine transporter } \\ \text { FOV } & \text { Field of view } \\ \text { PD } & \text { Parkinson's disease } \\ \text { ROI } & \text { Region of interest } \\ \text { SN } & \text { Substantia nigra } \\ \text { VOI } & \text { Volume of interest }\end{array}$

\section{Introduction}

Parkinson's disease (PD) is a progressive neurodegenerative disorder characterized by a severe loss of nigrostriatal neurons resulting in dopamine depletion in the nigrostriatal circuitry. Although the majority of underlying genetical and molecular causes are unknown, idiopathic PD has been attributed to environmental factors such as toxins causing oxidative stress in the dopaminergic neurons. This understanding has lead to the creation of several animal models 
mimicking the symptoms of PD. The macaque model after systemic MPTP administration [1] or the 6-hydroxydopamine (6OHDA) rat model [2] are currently the best documented but are also very severe and not-or shortlyprogressive PD models [3]. It is only recently that genes associated with familial forms of PD have been discovered [4], leading already to alternative rodent models based on local lentiviral vector induction [5-9] and transgenic models. These new chronic models will provide the opportunity to study the onset and further course of PD-related pathology at molecular, anatomical and behavioral level.

Instead of traditionally used invasive techniques in the characterization of animal models such as histology and autoradiography, non-invasive in vivo imaging is offering opportunities such as longitudinal studies to investigate the course of a neurodegenerative disorder [5] and to evaluate putative therapies; both are only about to be explored in rodent models. Few MRI studies on rodent models of PD exist [10-14], but the results are often hard to compare due to the use of different models, imaging protocols or field strengths and no general overview on observed MR contrast changes currently exists. It is, however, important to explore the possibilities of MRI and define contrast differences in a robust and well known animal model — where the degree of neurodegeneration can be experimentally controlled by the concentration of the neurotoxin - which then can be used as a reference standard for transgenic models.

In the present study, we used the 6OHDA rat model of PD where unilateral injection of 6OHDA in the substantia nigra results in destruction of more than $90 \%$ of the dopaminergic neurons. As a biological analog of dopamine and noradrenaline, 6OHDA is transported into catecholaminergic neurons, where it is metabolized, causing cytotoxicity and subsequent neuronal cell death [15]. The validity of this animal model has been demonstrated by invasive histological and electrophysiological analysis [15] and by in vivo imaging techniques such as microSPECT (single photon emission computed tomography) consistently demonstrating large decreases in DATbinding capacity in the affected striatum [16-19].

Here we describe asymmetric changes of different MR contrast features in the brain, which are not always due to changes in the affected hemisphere and therefore rather imply compensatory mechanisms in the intact hemisphere, which were suggested earlier in this model by electrophysiological studies, extracellular dopamine concentrations and receptor sensitivity [15].

\section{Material and methods}

Animal model

In total 12 healthy female Wistar rats $(282 \pm 12 \mathrm{~g})$ were submitted to the following experimental protocols (six 6OHDA rats and six controls). All aspects of animal experiments and husbandry were carried out in compliance with national and European regulations and were approved by the Animal Care and Use Committees of the Universities of Antwerp or Leuven.

\section{Surgery}

To avoid surgical artifacts in the region of interest (ROI: striatum),we have chosen unilateral stereotaxic injection in the (right) substantia nigra of $24 \mu \mathrm{g}$ 6OHDA (Sigma, St. Quentin-Fallavier, France) dissolved in $3 \mu 1$ of sterile $\mathrm{NaCl}$ solution containing $0.1 \%$ ascorbic acid.

All surgical aseptic procedures were performed under ketamine $(60 \mathrm{mg} / \mathrm{kg}$ i.p.) and medetomidine $(0.4 \mathrm{mg} / \mathrm{kg})$ anesthesia. Six rats were placed in a stereotaxic head frame (Stoelting, Wood Dale, Illinois) and a small hole was drilled in the skull in the appropriate location using bregma as reference. The coordinates used to target the substantia nigra were anterio-posterior (AP) $-5.2 \mathrm{~mm}$, lateral (L) $2.3 \mathrm{~mm}$ and dorsoventral (DV) $7.2 \mathrm{~mm}$. After the injection, the needle was left in place for an additional $10 \mathrm{~min}$ before being slowly retracted from the brain. No surgery was performed on control animals.

\section{Behavioral testing}

Amphetamine not only inhibits dopamine reuptake through direct dopamine transporter (DAT) binding, but also stimulates dopamine release through DAT. Systemic administration of amphetamine after unilateral dopaminergic lesioning results in ipsiversive turning behavior, where the number of rotations is related to the extent of the lesion [20-22]. Turning behavior of more than 100 ipsiversive rotations in $90 \mathrm{~min}$ correlates with a successful and detectable lesion in the substantia nigra. In our study, amphetamine-induced asymmetric rotational behavior was monitored in the first three control and three 6OHDA-lesioned rats after imaging using an automated rotometer bowl [23]. Amphetamine was injected intraperitoneally at a dose of $5 \mathrm{mg} / \mathrm{kg}$. For each test, the total number of complete turns clockwise and anti-clockwise was counted over $90 \mathrm{~min}$. The direction ipsilateral to the lesion is considered as positive. The analysis of amphetamine tests was based on net ipsilateral turns (defined as clockwise turning in the case of a right-sided injection).

\section{Anesthesia and monitoring}

MRI Rats were initially anesthetized with $5 \%$ isoflurane administered in a gaseous mixture of $30-70 \% \quad \mathrm{O}_{2}-\mathrm{N}_{2}$. 
Anesthesia was continued under $1.5 \%$ isoflurane presented to the rats via a mask fitting on the rat snout. Rats were breathing spontaneously throughout the entire experiment. Respiration rate, measured with a small pneumatic pillow, and body temperature, measured with a rectal probe, were continuously monitored with pcsam software (SA Instrument rents, NY, USA). Body temperature was kept constant at $37.5 \pm 0.5^{\circ} \mathrm{C}$ with a control heated warm water blanket (T/Pad and T/Pump, Gaymar Institute, Kent Scientific Corporation, Litchfield, CT). A tail vein was catheterized for administration of Dotarem (Guerbet, France) during perfusion measurements. The rats were allowed to breath spontaneously during the entire experiment. End tidal $\mathrm{CO}_{2}$ measurements and breathing rate were measured with a CapStar-100 End-Tidal $\mathrm{CO}_{2}$ Analyser (Linton instruments, Palgrave, UK). Breathing rates and end tidal $\mathrm{pCO}_{2}$ remained constant and normal during the entire recording period.

MicroSPECT Since isoflurane interacts with DAT [24,25], the barbiturate Nembutal ${ }^{\circledR}$ (natrium pentobarbital-Ceva Sante Animale, Brussels, Belgium) was used as anesthetic. Anesthesia was induced with a $60 \mathrm{mg} / \mathrm{kg}$ intraperitoneal injection. Five minutes later a tail vein was catheterized for administration of $300 \mathrm{MBq}$ of ${ }^{123}$ I-FPCIT ( ${ }^{123}$ I-ioflupane, DaTSCAN $^{\mathrm{TM}}$, GE Healthcare, England). Specific activity of the administered radioligand was $2,5-4,510^{14} \mathrm{~Bq} / \mathrm{mmol}$. SPECT imaging was started about $90 \mathrm{~min}$ after injection.

\section{Imaging}

\section{Uniform restrainer}

Imaging started approximately 4 weeks after surgery for the 6OHDA rats and was performed in age matched control animals. A stereotaxic device equipped with blunt ear plugs and a tooth bar incorporating the snout mask to avoid any head movement was developed according to Scherfler [18]. This allowed a uniform restraining of the rat's head in flat skull position in both imaging modalities (MRI and microSPECT). MRI measurements followed microSPECT measurements after 1-2 weeks.

\section{MRI}

MRI was performed on a $7 \mathrm{~T}$ (MRRS, UK) MR system with self-shielded gradients of $400 \mathrm{mT} / \mathrm{m}$ and aperture of $8 \mathrm{~cm}$ (Oxford instruments). A 50- mm Helmholtz transmitting and 24- $\mathrm{mm}$ receiving surface coil was fixed on the stereotaxic device, such that the centers of the coils were approximately at the position of bregma.

$\mathrm{T}_{2}$ - and $\mathrm{T}_{1}$-weighted high-resolution imaging of the entire rat brain (Field of view $(\mathrm{FOV})$ : $(35 \times 35 \times 30) \mathrm{mm}^{3}$, slice orientation: coronal) was performed by a three dimensional (3D)
Fast Spin Echo (FSE) (acquisition matrix: $256 \times 128 \times 64$; $\mathrm{TR}=2,500 \mathrm{~ms}$, effective $\mathrm{TE}=35 \mathrm{~ms}$, echo train length $=$ 4) and a 3D conventional SE (acquisition matrix: $256 \times 128 \times$ 128; $\mathrm{TR} / \mathrm{TE}=298 / 24 \mathrm{~ms}$ ) sequence.

In order to obtain quantitative information for the tissue $\mathrm{T}_{2}$ within the striatum, a SE multi-slice (FOV: $30 \mathrm{~mm}$; 12 horizontal slices covering the entire brain; slice thickness: $1 \mathrm{~mm}$ ) multi-experiment was performed (acquisition matrix: $256 \times 128 ; \mathrm{TR}=1,920 \mathrm{~ms}$ ). This included three experiments where the $\mathrm{T}_{2}$ weighting was gradually increased $\left(\mathrm{TE}_{1}=18 \mathrm{~ms}, \mathrm{TE}_{2}=36 \mathrm{~ms}, \mathrm{TE}_{3}=50 \mathrm{~ms}\right)$. At the same position and orientation of the $\mathrm{T}_{2}$-weighted images, $\mathrm{T}_{1}$ weighted multi-slice imaging was performed with an inversion recovery SE sequence (FOV: $30 \mathrm{~mm}$; slice thickness: $1 \mathrm{~mm}$; acquisition matrix $128 \times 64 ; \mathrm{TR}=10 \mathrm{~s}, \mathrm{TE}=20 \mathrm{~ms}$ ) by increasing inversion times $\left(\mathrm{TI}_{1}=200 \mathrm{~ms}, \mathrm{TI}_{2}=600 \mathrm{~ms}\right.$, $\mathrm{TI}_{3}=1,200 \mathrm{~ms}, \mathrm{TI}_{4}=1,800 \mathrm{~ms}, \mathrm{TI}_{5}=2,400 \mathrm{~ms}$, $\mathrm{TI}_{6}=3,000 \mathrm{~ms}, \mathrm{TI}_{7}=4,600 \mathrm{~ms}, \mathrm{TI}_{8}=6,200 \mathrm{~ms}, \mathrm{TI}_{9}=$ $8,000 \mathrm{~ms}$ ).

Bolus tracking was performed applying a single slice GE EPI at the position of the striatum (coronal slice, IA $8.5 \mathrm{~mm}$; FOV: $35 \mathrm{~mm}, \mathrm{TR}=300 \mathrm{~ms}, \mathrm{TE}=12 \mathrm{~ms}$, acquisition matrix $64 \times 64)$. Two hundred experiments were recorded and a bolus (Gadoterate meglumine, Gd-DOTA, Dotarem, Guerbet, France Dotarem, $0.2 \mathrm{mmol} / \mathrm{kg}$ ) was injected manually at the 50th experiment.

\section{MicroSPECT}

MicroSPECT was performed on either a single head General Electric Millennium MPR gamma camera (GE Healthcare, US) with a vendor-supplied single pinhole collimator or using a single head of a dual-head Siemens E-Cam camera (Siemens, Erlangen, Germany) with an in-house built collimator. Both systems have a single 3-mm knife-edge pinhole aperture. The focal length is $240 \mathrm{~mm}$ (Millennium) and $170 \mathrm{~mm}$ (E-Cam), respectively. Image acquisition consisted of 64 equidistant projections over $360^{\circ}$ with the pinhole aperture on a $4-\mathrm{cm}$ (E-Cam) or 5-cm (Millennium) radius of rotation. The projections were measured with a $15 \%$ energy window around the $159-\mathrm{keV}$ peak of ${ }^{123} \mathrm{I}$. Projections were rebinned in a $128 \times 128$ projection matrix with a pixel size of $1.695 \mathrm{~mm}$ for the Millennium system and a $256 \times 256$ matrix with $1.948 \mathrm{~mm}$ pixel size for the E-Cam system. On each camera system, three control and three 60HDA rats were measured so there was no camera bias. For reconstruction calibration purposes, the pinhole acquisition geometry was determined by an in-house developed method [26,27].

\section{Histology}

To assess degeneration of the nigrostriatal neurons, deep Nembutal ${ }^{\circledR}$ (natrium pentobarbital $60 \mathrm{mg} / \mathrm{kg}$ ) anesthesia was 
applied and then the animals were perfused transcardially with saline followed by ice-cold $4 \%$ paraformaldehyde in phosphate buffered saline (PBS) for $15 \mathrm{~min}$ after the last MRI measurement. The brain was postfixed overnight in the same fixing solution. $50 \mu \mathrm{m}$-thick coronal brain sections were cut with a vibratome (Leica, Microsystems, Wetzelar, Germany) and stored at $4{ }^{\circ} \mathrm{C}$. First, sections were treated with 3\% hydrogen peroxide and incubated overnight with rabbit anti-tyrosine hydroxylase (1:5000) (Chemicon, Temecula, CA) in $10 \%$ normal swine serum. Then the sections were incubated in biotinylated swine anti-rabbit secondary antibody, followed by incubation with Strept-ABC-HRP complex (DAKO, Glostrup, Denmark). Detection was by diaminobenzidine (DAB) using $\mathrm{H}_{2} \mathrm{O}_{2}$ as a substrate.

\section{Data processing}

\section{$M R I$}

Reconstruction MR data were Fourier transformed to image matrices of $(256 \times 256 \times 256),(256 \times 256)$ or $(128 \times 128)$ for the $3 \mathrm{D}$ high resolution images, $2 \mathrm{D} \mathrm{T}_{2}$ - and $2 \mathrm{D} \mathrm{T}_{1}$ - weighted $\mathrm{SE}$, and $\mathrm{T}_{2}{ }^{*}$ - weighted GE EPI images respectively. This resulted in an in plane resolution of 137, 117, 234 and $273 \mu \mathrm{m}$ for the $3 \mathrm{D}$ high-resolution, $\mathrm{T}_{2}$ - and $\mathrm{T}_{1}$ - weighted, and GE EPI images, respectively.

MR image processing The calculation of quantitative maps in which the signal intensity is proportional to the tissue $T_{1}$ or $\mathrm{T}_{2}$ value was carried out using in-house software developed in IDL (Interactive Data Language). $\mathrm{T}_{1}$ and $\mathrm{T}_{2}$ values were measured separately in left and right striatum in three brain slices at positions IA $4-\mathrm{IA} 6 \mathrm{~mm}$, where the structure is clearly delineable based on anatomical boundaries.

The $T_{2}$ values were calculated on a pixel-by-pixel basis. The signal intensities of the three SE images with different TE were plotted versus echo time and then the best fit to the exponential equation describing the $\mathrm{T}_{2}$-relaxation process of the spin system was determined:

$S_{i}=S_{0} \exp \left(-\mathrm{TE}_{i} / T_{2}\right)$

with $S_{i}$ being the pixel signal intensity of the SE image acquired with an echo time $\mathrm{TE}_{i}$ as defined in the acquisition method previously. This pixel-by-pixel fitting resulted in $T_{2}$-maps, images in which the gray level of each pixel represents the fit-parameter $\mathrm{T}_{2}$.

Analogous to the $T_{2}$ mapping, the $T_{1}$ values were calculated on a pixel-by-pixel basis. The signal intensities of the nine IR-SE images were fitted to the inversion time following the general $\mathrm{T}_{1}$-relaxation process determined by:
$( \pm) S_{i}=S_{0}\left(A-B \exp \left(-\mathrm{TI}_{i} / T_{1}\right)\right)$

with $( \pm) S_{i}$ the (inverted or non-inverted) pixel signal intensity of the IR-SE image acquired with an inversion time $\mathrm{TI}_{\mathrm{i}}$ as defined in the acquisition method above. To deal with the needed negative values for the short inversion times, we first searched the $\mathrm{TI}_{\min }$, which has minimal SI. Then, the fitting procedure was repeated 5 times in which a different number of $S_{i}$ were inverted for the shortest inversion times (symmetric around $\mathrm{TI}_{\min }$ ). The $\mathrm{T}_{1}$ value was defined by the fit that showed the smallest deviation from the measured SIdata. This pixel-by-pixel fitting resulted in $\mathrm{T}_{1}$-maps, images in which the gray level of each pixel represents the fit-parameter $\mathrm{T}_{1}$.

Bolus tracking MR data were transferred to a Linux work station and analyzed using Medx Software (Medx, Sensor Systems, Sterling, USA). The dynamic images were viewed as mean intensities on a pixel-per-pixel basis to display susceptibility curves. The signal intensity-versus-time curve was normalized to baseline scans before bolus passage. The observed change in the relaxation rate $\mathrm{R}_{2}^{*}\left(=1 / \mathrm{T}_{2}^{*}\right)$ is approximately proportional to contrast concentration in the tissue. This change in relaxation rate $\left(\Delta R_{2}^{*}\right)$ for each time point $t$ can be obtained from the change in signal intensity after contrast administration $(\mathrm{S}(\mathrm{t}))$ from the baseline signal before contrast administration $\left(\mathrm{S}_{0}\right), \Delta \mathrm{R}_{2}^{*}=-\ln \left(\mathrm{S}(\mathrm{t}) / \mathrm{S}_{0}\right) / \mathrm{TE}$. Effects of recirculation of the contrast are reduced by numerically integrating the $\Delta \mathrm{R}_{2}^{*}$-versus-time curve according to a gammavariate fitting curve. An improved gamma-variate fit was used to reduce underestimation of the arterial input function, which otherwise leads to overestimation of cerebral blood volume (CBV) and flow (CBF) values. The arterial input function was calculated from an artery likelihood map representing voxels within the brain that are ranked based on the likelihood of being an artery. Arterial voxels were defined by following criteria: large peak height, small peak width, early take-off time and steep initial slope. The arterial likelihood map was based on maximizing: $P$ (early takeoff) $* P$ (narrow peak-width)* $P($ large peak-height $)$, where $P$ means probability. Parametric images displaying CBV and $\mathrm{CBF}$ on a pixel-per-pixel base were used for analysis. From these perfusion maps, arterial voxels were removed such that only the parenchymal contribution was reflected and hence less variation in the $\mathrm{CBV}$ and $\mathrm{CBF}$ values of the region of interest was observed. Mean values of the quantitative parameters were determined in both left (intact) and right (affected) striatum.

Asymmetry ratios between ipsi- (right) and contralateral (left) striatum were calculated for all parameters to define intraanimal differences (differences within the striatum of animals from the same group) by dividing ipsilateral value with contralateral value, or for control animals right divided by left. 


\section{MicroSPECT}

Reconstruction Iterative OS-EM image reconstruction, incorporating the camera calibration, was performed in a $64 \times 64 \times 80$ image with a pixel size of $1.2 \mathrm{~mm}$. The reconstruction used an iteration scheme with 10 iterations of a decreasing number of subsets $[2,4,8,12,16]$ and [1], yielding 430 equivalent MLEM iterations. The full reconstruction methodology has been described elsewhere [26]. In summary, the reconstruction takes the pinhole sensitivity into account and also models the pinhole spatial resolution by a multiple ray tracing technique (7 rays were used).

Quantification DAT-binding indices were calculated as activity in the striatum divided by aspecific activity in the cerebellum minus 1 , giving an indicator of DAT-binding capacity (or distribution volume ratio DVR). Volumes of interest (VOI) for the putamen that were delineated bilaterally and for the cerebellum were used for automated activity quantification. Left-to-right binding asymmetry indices were determined [28].

\section{Quantitative histology}

The number of TH-positive neurons in the SN pars compacta was estimated in both control and lesion side using an unbiased stereological method: the optical fractionator (Stereo Investigator, MicroBrightField, Williston, VT, US). Every fifth section throughout the substantia nigra was analyzed with a total of 7-8 sections for each animal. Lesion size was expressed as the percentage of neuronal loss measured in the lesioned side when compared to total number of nigrostriatal neurons in the control side, where no neuronal loss is assumed.

\section{Statistical analysis}

After testing for normal distribution, parametric tests were applied for all statistical analyses, using SPSS 12.0 (Windows). Significance was accepted at the $95 \%$ probability level, and all reported values of the present study are mean \pm standard error of the mean.

Inter-animal differences for all MRI and SPECT parameters measured in the right striatum of respectively control when compared to 6OHDA-lesioned animals, were calculated using two-tailed, independent, sample it t-tests. Intra-animal differences for MRI and SPECT parameters, measured in left when compared to right striatum of control or 6OHDA-lesioned animals were calculated by twotailed, paired, $t$-tests. Alternatively, intra-animal differences for MRI and SPECT parameters were also expressed as asymmetry ratios that were statistically compared to 1 using a one sample $t$-test.

\section{Results}

Validation of the 6-hydroxydopamine model

\section{Behavioral measurements}

Of six 6OHDA rats, three were subjected to the behavioral tests and displayed 409, 1,167 and 1,661 ipsiversive rotations, indicating that the site of injection and injection dose were sufficient to cause a (near-) complete lesion in the substantia nigra (Table 1). In three control animals, ipsiversive rotations of $-133,-52$ and 4 were counted.

\section{$M R I$}

In some rats, a lesion at the level of the SN was still clearly visible 4 weeks after injection. This lesion was restricted to the ipsilateral SN and did not extend to the contralateral SN nor the ipsilateral striatum.

\section{DAT binding}

Figure 1a and Table 1 show the binding capacity of the DAT in the left and right striatum of control animals when compared to 6OHDA rats. While in control animals there was no difference of DAT binding in left or right striatum (binding asymmetry ratio $1.01 \pm 0.04, p=0.7), 6 \mathrm{OHDA}$ rats showed a significant decrease in the affected striatum $(0.26 \pm$ $0.09, p<0.001$ ) (Fig. 1b). This was also significant when compared with control animals ( $p<0.001$ ). In one 6OHDA rat, binding capacity was only reduced by $35 \%$ while in the other animals a 70-93\% decrease was noted. The binding capacity of DAT in the unaffected striatum of 6OHDA rats showed higher values when compared to the left striatum of control animals, but this difference did not reach significance $(0.89 \pm 0.07$ vs. $0.74 \pm 0.09, p=0.2)$.

\section{Histology}

Three animals were sacrificed for histological analysis. In the substantia nigra, every fifth section of the brain was stained for tyrosine hydroxylase (TH) and quantified by stereological procedures. An average loss of $97 \pm 0.6 \%$ neurons was observed in the substantia nigra (Fig. 2; Table 1).

\section{Quantification of MRI parameters}

Left and right striatum were separately delineated as was done in the $\mu$ SPECT analysis (see ROI 1 and 2 in Fig. 3). 
Table 1 A summary of the results (means \pm SE) of the multi-modality study on the 6OHDA- lesioned $(n=6)$ and control animals $(n=6)$

\begin{tabular}{|c|c|c|c|c|c|c|}
\hline & \multicolumn{3}{|l|}{ Control } & \multicolumn{3}{|l|}{ 6OHDA } \\
\hline & Left & Right & Asymmetry ratio & Left & Right & Asymmetry ratic \\
\hline \multicolumn{7}{|l|}{ MRI parameters } \\
\hline $\mathrm{T}_{2}(\mathrm{~ms})$ & $42.09 \pm 0.58$ & $40.73 \pm 0.68$ & $0.97 \pm 0.02$ & $42.71 \pm 0.81$ & $40.98 \pm 0.84$ & $0.96 \pm 0.02^{\mathrm{a}}$ \\
\hline $\mathrm{T}_{1}(\mathrm{~ms})$ & $1,668 \pm 26$ & $1,681 \pm 18$ & $1.01 \pm 0.02$ & $1,593 \pm 48$ & $1,699 \pm 55$ & $1.07 \pm 0.02^{\mathrm{a}}$ \\
\hline $\mathrm{CBF}(\mathrm{ml} / 100 \mathrm{~g} / \mathrm{min})$ & $257.1 \pm 72.9$ & $257.7 \pm 71.7$ & $1.02 \pm 0.01$ & $761.0 \pm 26.0$ & $583.3 \pm 31.6$ & $0.8 \pm 0.04^{\mathrm{a}}$ \\
\hline CBV (ml/100g) & $4.4 \pm 0.2$ & $4.4 \pm 0.2$ & $1.00 \pm 0.02$ & $6.2 \pm 1.2$ & $5.0 \pm 0.8$ & $0.8 \pm 0.05^{\mathrm{a}}$ \\
\hline$\mu$ SPECT DAT binding & $0.74 \pm 0.09$ & $0.74 \pm 0.09$ & $1.01 \pm 0.04$ & $0.89 \pm 0.07$ & $0.23 \pm 0.07$ & $0.26 \pm 0.09^{\mathrm{a}}$ \\
\hline $\begin{array}{l}\text { Behavior }\left(\mathrm{n}_{\text {controls }}=3\right. \\
\left.\mathrm{n}_{6 \mathrm{OHDA}}=3\right)\end{array}$ & $-60.3 \pm 39.8$ & & & $1,079 \pm 511$ & & \\
\hline Histology $\left(\mathrm{n}_{6 \mathrm{OHDA}}=3\right)$ & ND & & & $97.0 \pm 0.6 \%$ & & \\
\hline
\end{tabular}

Behavior data point out to number of ipsiversive rotations as measured after amphetamine administration. Histology refers to the lesion size or neuronal loss measured in the ipsilateral SN. $C B F$ cerebral blood flow, $C B V$ cerebral blood volume, $D A T$ dopamine transporter, $N D$ not done, $\mathrm{TH}$ tyrosine hydroxylase

${ }^{a}$ Asymmetry ratios significantly different from $1(p<0.05)$

\section{MRI relaxation parameters}

Although both control and 6OHDA animals seemed to show an asymmetry in $T_{2}$ values between ipsi- and contralateral striatum, only for 6OHDA rats the difference between intact (contralateral) and affected (ipsilateral) striatum was significant $\left(p_{\mathrm{T} 2-\text { control }}=0.07, p_{\mathrm{T} 2-6 \mathrm{OHDA}}=0.04\right)$ (Fig. 3). After calculation of the $\mathrm{T}_{2}$ asymmetry according to Hall and colleagues [29] as the ratio of the $T_{2}$ measured in the ipsilateral versus contralateral striatum, an asymmetry significantly different from 1 could only be discerned in the 6OHDA group $(p=0.04)$, but not in the control group $(p=0.08)$, discriminating 6OHDA rats from control animals by a single measurement.

In contrast to control animals where there was no difference for $\mathrm{T}_{1}$ between left and right striatum $\left(p_{\mathrm{T} 1-\text { control }}=\right.$ $0.47), T_{1}$ in the affected striatum of 6OHDA- lesioned rats was significantly different from that in the intact hemisphere $\left(p_{\mathrm{T} 1-6 \mathrm{OHDA}}=0.005\right)$ (Fig. 3$)$. In addition, the $\mathrm{T}_{1}$ asymmetry ratio of the 6OHDA rats was not only significantly different from 1 ( $p=0.005)$, but was also significantly increased by $5 \%$ when compared to control animals (controls: $1.01 \pm 0.02$; 6OHDA: $1.07 \pm 0.02 ; p=0.04$ ) (Fig. 3). For control animals, the asymmetry ratio was not different from $1(p=0.45)$.

\section{Changes in cerebral blood flow and volume}

Dynamic contrast-enhanced MRI, or bolus tracking, was used to investigate alterations in the resting state cerebral blood volume and flow. Though in the previous experiments horizontal slices were preferred for an optimal discrimination of the striatum from the surrounding tissue, this slice orientation was shown to suffer from too many susceptibility artifacts in fast imaging sequences. Therefore, hemodynamic
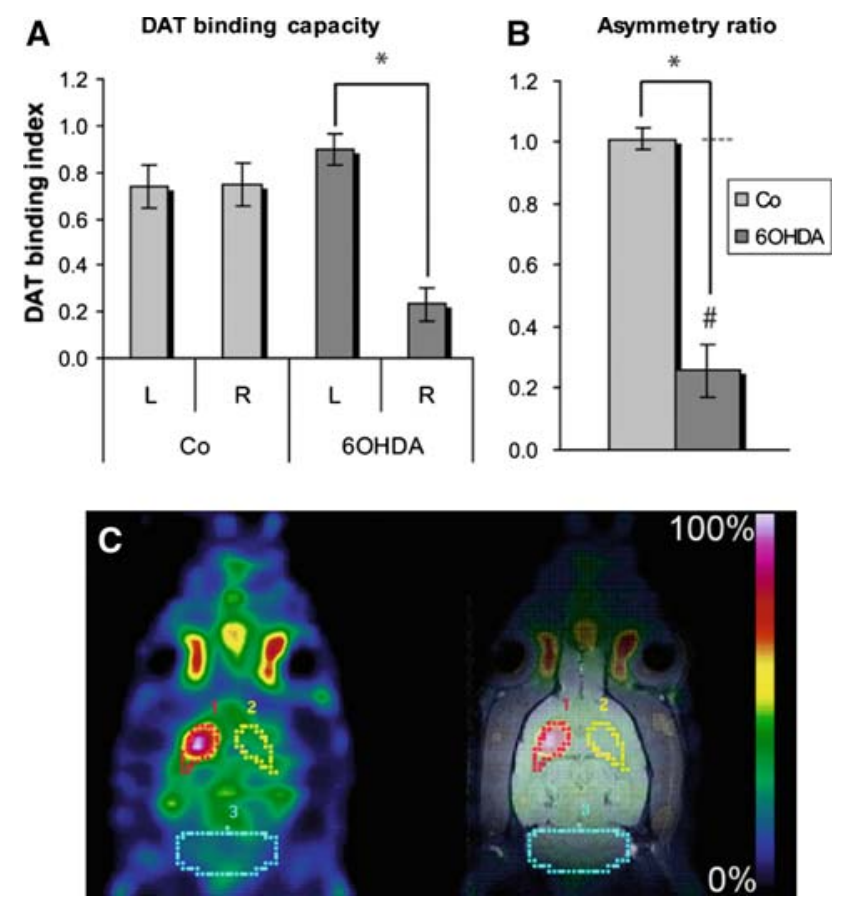

Fig. 1 a The dopamine transporter (DAT) binding capacity in left (L) and right $(R)$ striatum of control $(\mathrm{Co})$ animals and 6OHDA-lesioned rats. The affected striatum of 6OHDA rats displayed a significant decreased DAT-binding capacity $(*, p<0.001)$. b Right-left asymmetry ratio of DAT-binding capacity. Double crosses (\#) show significant difference $p<0.05$ ) from the test value 1 indicated with the dashed line. Asymmetry ratio is significantly different $(p<0.001)$ between control and 6OHDA rats $(*)$. $\mathbf{c}$ Image fusion display of regionof-interest placement (Hermes Multi modality Display, Nuclear Diagnostics, Stockholm, Sweden). Specific binding activity of DAT was measured in left (1) and right (2) striatum and non-specific activity in the cerebellum (3)

parameters were measured in a single coronal slice at position IA $8.5 \mathrm{~mm}$.

Control animals did not display any intra-animal differences in the $\mathrm{CBF}$ nor $\mathrm{CBV}$ values between left and right 


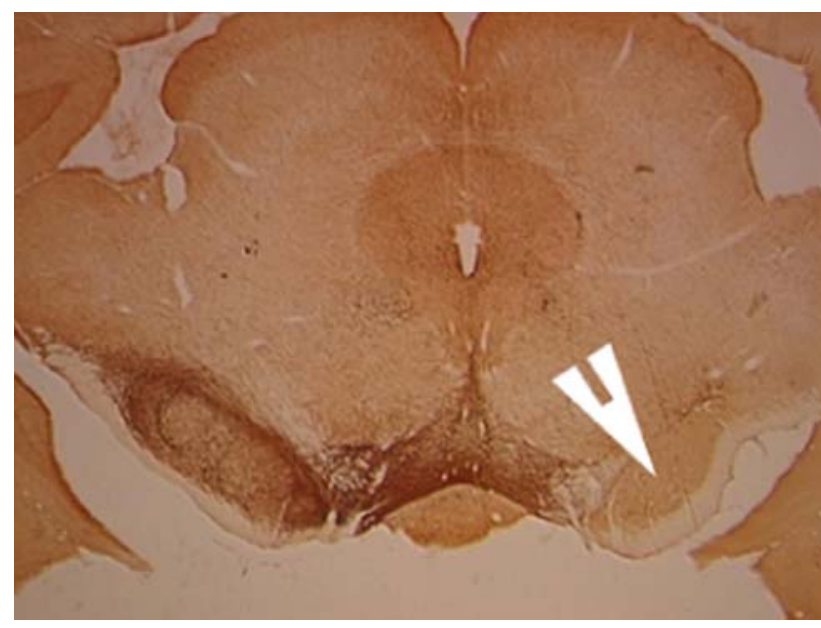

Fig. 2 Tyrosine hydroxylase staining (right) shows no reaction at the 6OHDA injection side (right). Histological quantification revealed an average loss of $97 \pm 0.6 \%$ of the neurons within the affected substantia nigra (arrowhead)

striatum $\left(p_{\mathrm{CBF}}=0.3, p_{\mathrm{CBV}}=0.98\right)$, neither were the rightleft asymmetry ratios significantly different from $1\left(p_{\mathrm{CBF}}=\right.$ $\left.0.7, p_{\mathrm{CBV}}=0.9\right)(\mathrm{Fig}$. 4a-c). This is in contrast to 6OHDAlesioned rats in which a significant intra-animal difference was shown between left and right striatum for both $\mathrm{CBF}$ ( $p=0.008$, Fig. 4a) and CBV ( $p=0.006$, Fig. 4b), which resulted in an asymmetry ratio significantly different from 1 $\left(p_{\mathrm{CBF}}=0.02, p_{C B V}=0.02\right.$, Fig. $\left.4 \mathrm{c}\right) . \mathrm{CBF}$ in both intact and affected striatum were significantly increased when compared to control animals. CBV values tended to be increased especially in the intact striatum, however, they did not reach significance (Fig. 4a).

In summary, it was shown that hemodynamic parameters displayed a clear asymmetry in 6OHDA rats, which was reflected in an asymmetry ratio significantly different from 1. Correlation studies were performed between different data sets, but no significance could be demonstrated. All values are summarized in Table 1.
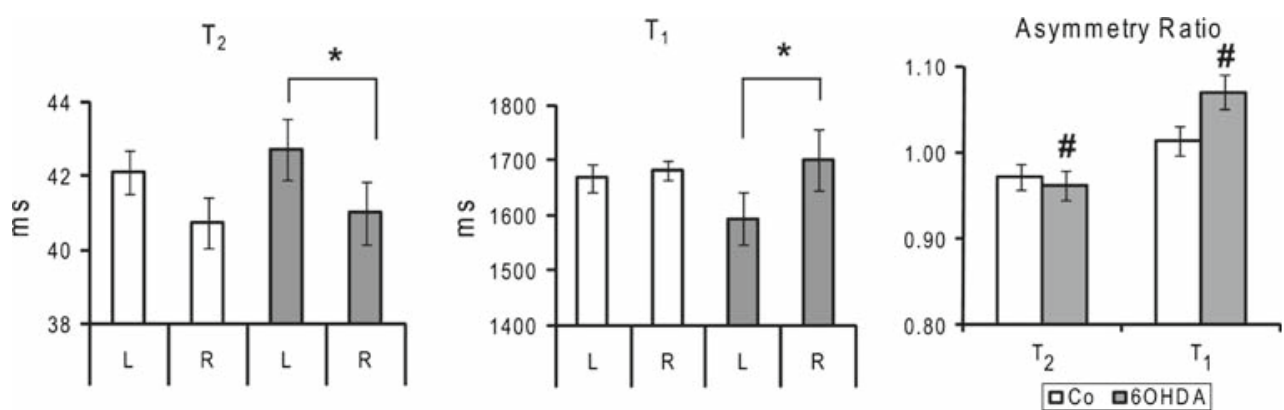

Fig. 3 Relaxation rates $\left(\mathrm{T}_{2}\right.$ and $\left.\mathrm{T}_{1}\right)$ measured in left $(L)$ and right $(R)$ striatum of control (Co) and 6OHDA rats. Asterisk (*) shows significant difference between left and right striatum for $\mathrm{T}_{2}$ and $\mathrm{T}_{1}$. Double crosses
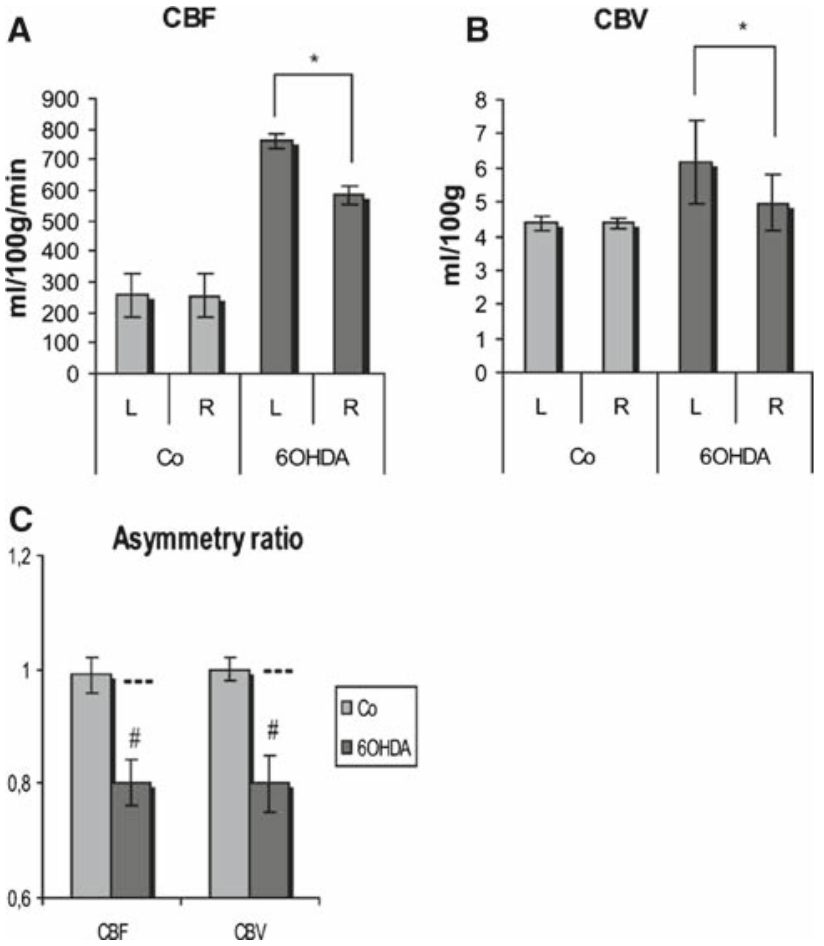

Fig. 4 Column charts demonstrating the changes in cerebral blood flow (a) and volume (b) measured in left $(L)$ and right $(R)$ striatum of control (Co) and 6OHDA rats. Asterisk $(*)$ shows significant difference between left and right striatum for CBV and CBF. c Right-left asymmetry ratio of CBV and CBF. Double crosses (\#) show significant differences $(p<0.05)$ from the test value 1 indicated with the dashed line

\section{Discussion}

The cardinal neurological symptoms of Parkinson's disease only occur if more than $80 \%$ of the nigrostriatal pathway is degenerated. Before, compensatory mechanisms occur in the remaining intact basal-ganglia cortical circuitry masking the degeneration of the nigrostrial neurons. Unilateral injection of 6OHDA in the substantia nigra (SN) of the rat causes an acute and nearly complete lesion at the injection site, resulting in dopamine (DA) depletion in only the ipsi-

\footnotetext{
(\#) indicate right-left asymmetry ratio of relaxation rates significantly $(p<0.05)$ smaller than the test value 1 . Asymmetry ratio of $\mathrm{T}_{1}$ is significantly different $(p<0.05)$ between control and 6OHDA rats $(*)$
} 
lateral striatum. Most studies focus on changes observed in the ipsilateral hemisphere, while it is known that also in this model compensatory mechanisms take place contralaterally [3]. Here we studied the 6OHDA model for PD using microSPECT and MRI, measuring all parameters separately in left and right striatum of 6OHDA rats and controls in order to evaluate not only differences in the ipsilateral, but also compensatory mechanisms in the contralateral striatum.

Animal model

The lesion extent in the 6OHDA group was validated by behavioral, $\mu$ SPECT and finally histological studies. Unilateral lesions cause an asymmetric and quantifiable motor behavior, such as rotational behavior that occurs when striatal dopamine loss exceeds $70 \%$. The number of ipsiversive turnings can be correlated with the degree of cell loss in the $\mathrm{SN}$. In the present study, ipsiversive turning in the amphetamine challenge test was by average larger than 500, which is known to correlate with massive lesions. This was further confirmed by $\mu$ SPECT imaging revealing a decreased DAT- binding capacity in the striatum by almost $74 \pm 8 \%$, which was related to a neuronal loss in the substantia nigra of $97 \pm 1 \%$ as shown by histological findings.

Previously, Kondoh and colleagues [30] assessed the effective dose of 6OHDA by $\mathrm{T}_{2}$-weighted imaging one day after injection, and suggested an effective dose lower than the concentration 6OHDA injected in the present study. Nevertheless, the current protocol has previously been used by these authors [5] showing effective lesioning of the nigrostriatal pathway and resulting in complete dopamine depletion in the striatum. Though Kondoh [30] suggested non-specific lesioning in neighboring brain structures with higher doses, we observed a lesion that was confined to the ipsilateral SN 4 weeks after injection mimicking reliably the cardinal symptoms of Parkinson's Disease [31].

A major limitation of the present study is that no sham animals have been included. Sham animals are better controls compared to naive animals, as they undergo similar anesthetic and surgical procedures as the animal model under study. These experimental procedures, especially stereotaxic brain injections, might influence the obtained results. However, as our injection site ( $\mathrm{SNr}$ ) and $\mathrm{ROI}$ (striatum) are anatomically separated brain areas, we assumed that surgery would not affect measured values in the ROI. In addition, previous studies have shown that the functional differences between 6OHDA-lesioned rats and naive controls are unlikely to be related to experimental artifacts, such as the intranigral injection. Araujo and colleagues demonstrated that $\left[{ }^{18} \mathrm{~F}\right]$-FDG uptake, a measure for neuronal viability, in the injected region is similar between controls and sham-operated animals [32]. In addition, Kondoh and colleagues [30] examined the effect of 6OHDA and vehicle injection on $\mathrm{T}_{2}$ in the $\mathrm{SN}$ of rats one, three and nine days after injection. While vehicle injection did not cause any changes in $\mathrm{T}_{2}$, a hyperintense area was observed in $\mathrm{SNr}$ one and three days after 6OHDA injection Nine days after injection, $\mathrm{T}_{2}$ returned to basal values. Therefore, as we studied the morphological changes only in the projection area of the $\mathrm{SNr}$, we are confident that the comparison of our results to normal control animals is as valuable.

\section{MicroSPECT imaging}

MicroSPECT is a sensitive in vivo imaging technique allowing to quantify changes in DAT-binding capacity in the affected striatum, and thus is an in vivo tool to measure altered presynaptic nigrostriatal integrity [33]. In most animals, a severe reduction of DAT binding was noted indicating an appropriate nigrostriatal damage comparable to the loss observed in clinical Parkinson's disease. For quantification, the striatal volume of interest was determined on the corresponding MRI data sets and transformed by translations to the corresponding microSPECT image. In evaluating DAT loss quantitatively, we did not take into account partial volume effects in this study. This is likely to give an underestimation of the true impairment of striatal DAT-binding capacity. In future studies, voxel-based implicit partial volume correction schemes will be evaluated by incorporation of anatomy (based on MR images) in the reconstruction algorithm (A-MAP, Anatomical Maximum A Posteriori reconstruction) [34].

\section{MRI findings}

\section{Transversal relaxation rate, $T_{2}$}

$\mathrm{T}_{2}$-weighted MRI studies on patients with Parkinson's disease are performed to study the iron metabolism in the basal ganglia in an effort to discriminate patients from control subjects, but results are not consistent and even often contradictory. Very recently Kosta and colleagues [35] showed decreased $T_{2}$ values in the substantia nigra compacta, but higher $\mathrm{T}_{2}$ values in both nucleus caudatus and putamen, while Vymazal and colleagues [36] measured no difference in $T_{2}$ in the putamen and Ye et al. [37] reported an increased iron concentration. To our knowledge, only Hall and colleagues [29] measured $T_{2}$ changes in the striatum of the 6OHDA rat model for Parkinson's disease at $1.5 \mathrm{~T}$ to study the daily fluctuations of the iron metabolism. Although they were not 
able to discriminate 6OHDA-lesioned animals from controls by the asymmetry ratio, PD rats displayed higher daily fluctuations than the control or sham group. They suggested that this highly fluctuating iron metabolism underlies the contradicting results from the clinical studies. In addition to Hall and colleagues [29], we were able to reproduce the significant asymmetry ratios, and to discriminate controls from lesioned animals at a single time point. Within the 6OHDA group the absolute $T_{2}$ values were significantly smaller in the affected striatum when compared to the normal intact side. However, the comparison with control animals could not elucidate whether this significant asymmetry was due to decreased $\mathrm{T}_{2}$ values in the affected striatum or increased $\mathrm{T}_{2}$ in the normal striatum. While decreased $\mathrm{T}_{2}$ is related to iron accumulation, increased $\mathrm{T}_{2}$ values could be related to compensatory mechanisms such as sprouting of the remaining projecting fibers [35]. The resulting increased density of intact dopaminergic fibers containing a high amount of water could account for the increased $\mathrm{T}_{2}$ relaxation in the contralateral intact striatum. In an attempt to elaborate this hypothesis we performed Magnetization Transfer Contrast imaging in a subset of 6OHDA-lesioned rats and normal controls (data not shown). We were, however, not able to show significant asymmetry ratios. ${ }^{1} \mathrm{H}$ spectroscopy focusing on $\mathrm{N}$-acetylaspartate - a marker of neuronal/axonal integrity — might provide further insights in this hypothesis.

\section{Longitudinal relaxation rate, $T_{1}$}

Though the $\mathrm{T}_{1}$ asymmetry ratio of the 6OHDA group was significantly increased when compared to controls, it could not be elucidated whether this was attributed to increased $\mathrm{T}_{1}$ in the affected striatum or decreased $T_{1}$ in the normal appearing striatum. Increases in $\mathrm{T}_{1}$ can be related to several factors such as a decrease of lipid content, which is often observed in demyelinization disorders such as multiple sclerosis [38] but might also apply to the striatum of 6OHDA rats reflecting degeneration of the striae running through the grey matter. Caudate nucleus and putamen are in many mammals such as humans and primates well-defined striatal components and separated by the internal capsule, but are considered to form an indivisible complex in the rat-called the 'caudate-putamen'-due to the poor development of the internal capsule in rodents. Therefore, the fiber bundles of the internal capsule are scattered throughout the whole dorsal striatum of the rat brain, and hence preventing a myelo-architectonical separation of both subdivisions [39].

As inter-animal differences were never significant between control and 6OHDA-lesioned rats for $\mathrm{T}_{2}$ or $\mathrm{T}_{1}$, we were not able to explain to which mechanisms asymmetric changes can be attributed. Nevertheless, it was shown that at $7 \mathrm{~T}$ rats lesioned by 6 OHDA in the substantia nigra could be discriminated from control animals using the intra-animal asymmetry ratios of $T_{2}$ and $T_{1}$ measured in the striatum.

\section{Perfusion measurements}

Although the correlations between motor features and their cerebral substrates are not yet completely understood, a common expectation is that the alteration of functional activity in the basal ganglia in PD will be associated with changes in regional cerebral metabolism (rCMR) and regional cerebral blood flow (rCBF) in certain brain areas [40]. The classical, simplified view of basal ganglia connections is based upon two distinct striato-pallidal pathways: a 'direct' pathway leading from the striatum straight to the globus pallidus pars interna (GPI) and other basal ganglia output nuclei, while an 'indirect' pathway first transmits to the globus pallidus pars externa (GPE) and the STN. A prediction of the direct-indirect pathways model is that the akinesia associated with Parkinson's disease is due to an imbalance in activity in the two pathways in favor of the indirect pathway, resulting in an overactivity of basal ganglia outputs, which might explain the increased $\mathrm{CBF}$ we observed in the ipsilateral striatum [41]. Also in PD patients, increased CBF has been reported in the bilateral putamen, globus pallidum, thalamus and pons [40].

Remarkably, CBF was also significantly increased in the contralateral striatum of 6OHDA-lesioned animals. Electrophysiological recordings reported an increase in the number of spontaneously active striatal neurons after a dopaminergic neuronal loss of $75-85 \%$ that occurs in both striata and even more in the contralateral intact side [3]. In addition, it has been proposed that during early stages of illness, compensatory mechanisms including increased neuronal activity by residual dopaminergic neurons and increased input from contralateral circuits and structures outside the basal ganglia, may be active [42]. Yang and colleagues [41] studied cerebral blood flow in the 6-hydroxydopamine rat model five weeks after unilateral lesioning using autoradiography and showed that functional compensations occurred bilateral. In addition they showed that $\mathrm{CBF}$ was increased in the ipsilateral internal globus pallidus and decreased in the striatum and external globus pallidus. In a recent $\left[{ }^{18} \mathrm{~F}\right]-\mathrm{FDG}$ study in the same model 6-11 weeks after lesioning, it was shown that glucose metabolism was relatively increased in the contralateral midbrain comprising the substantia nigra [31]. Moreover, regional glucose metabolism was significantly lower in the ipsilateral caudate-putamen, nucleus accumbens, lateral globus pallidus, pons and hippocampus of 6OHDA-lesioned rats when compared to the contralateral hemisphere. These results question again the role of the intact striatum as internal control and the importance of control animals. 
Nevertheless, a large variability exists in the reported data on CBF measurements in 6OHDA-lesioned rats: two other studies have demonstrated no change in absolute CBF in the awake, restrained rat in the striatum 2 weeks after 6OHDA lesions of the left SN [43,44]; and another study performing lesions of the right SN and ventral tegmental area, found a decrease in CBF in the ipsilateral neostriatum and amygdala, but not in the cortex [45]. Such discrepancies may be ascribable to differences in the extent of lesion, the lesion sites or laterality.

Another possible underlying factor influencing $\mathrm{CBF}$ is the dopaminergic neurotransmission. Indeed, it has been suggested that dopamine exerts a direct effect on the vascular tone by dilatation of the small arterioles [46]. Asymmetric $\mathrm{CBV}$ values hence might reflect decreased extracellular DA levels in the affected striatum and increased DA levels in the contralateral striatum [3]. These results question again the role of the intact striatum as internal control and the importance of control animals.

\section{Conclusion}

It was shown that the asymmetry ratios of $\mathrm{T}_{1}, \mathrm{~T}_{2}$ and perfusion parameters appeared to be successful in discriminating 6OHDA rats from controls. As it is known that Parkinson's disease begins unilaterally in patients, a comparable observation might be expected in transgenic animals - mimicking the onset and progression of PD even more closely-and asymmetry ratios might be important parameters in future experimental studies in which e.g. the onset and further progression of the neurodegeneration is studied.

Though significant asymmetries might be obvious in a unilateral lesion model, we were not able to attribute the asymmetry ratios to ipsilateral changes only. We therefore refer to the plausible influence of compensatory mechanisms that are known to manifest such as axonal sprouting and increased dopamine turnover and which have been described in both the 6OHDA rat model [3] and patients [35]. Compensatory mechanisms also play an important role in preclinical PD patients as symptoms of Parkinson's disease do not appear until most dopaminergic projection neurons from $\mathrm{SN}$ to striatum are degenerated, implying that the remaining neurons compensate for the early neuronal loss in the asymptomatic stage. The results of this study question the role of the intact striatum as internal control in 6OHDA-lesioned animals and stress the importance of control animals.

Acknowledgments The assistance from Mr. P. Vermaelen, Ir. K. Vunckx, C. Casteels MSc and Dr. Pharm. B. Vanbilloen is greatly appreciated. JN and NVC are supported by Flemish Fund for Scientific Research (FWO Vlaanderen). VB is a postdoctoral fellow of the Flemish Fund for Scientific Research (G.0174.03, FWO Vlaanderen). KVL is supported by a Clinical Research Mandate of the Flemish Fund for Scientific Research. This work was supported by the Institute for the
Promotion of Innovation by Science and Technology in Flanders (IWT SBO/030238); and small projects BOF UA 2006. This research was carried out in the frame of the European-funded NoE EMIL (LSHC2004-503569) and DiMI (LSHB-CT-2005-512146). IUAP P6/38 2302.

\section{References}

1. Burns RS, Chiueh CC, Markey SP, Ebert MH, Jacobowitz DM, Kopin IJ (1983) A primate model of parkinsonism: selective destruction of dopaminergic neurons in the pars compacta of the substantia nigra by $N$-methyl-4-phenyl-1,2,3,6-tetrahydropyridine. Proc Natl Acad Sci USA 80(14):4546-4550

2. Mendez JS, Finn BW (1975) Use of 6-hydroxydopamine to create lesions in catecholamine neurons in rats. J Neurosurg 42(2):166173

3. Schwarting R, Huston J (1996) Unilateral 6-hydroxydopamine lesions of meso-striatal dopamine neurons and their physiological sequelae. Prog Neurobiol 49(3):215-266

4. Huang Y, Cheung L, Rowe D, Halliday G (2004) Genetic contributions to Parkinson's disease. Brain Res Brain Res Rev 46(1):44-70

5. Lauwers E, Beque D, Van Laere K, Nuyts J, Bormans G, Mortelmans L, Casteels C, Vercammen L, Bockstael O, Nuttin B, Debyser Z, Baekelandt V (2006) Non-invasive imaging of neuropathology in a rat model of alpha-synuclein overexpression. Neurobiol Aging 28(2):248-257

6. Lauwers E, Debyser Z, Van Dorpe J, De Strooper B, Nuttin B, Baekelandt V (2003) Neuropathology and neurodegeneration in rodent brain induced by lentiviral vector-mediated overexpression of alpha-synuclein. Brain Pathol 13(3):364-372

7. Baekelandt V, Claeys A, Eggermont K, Lauwers E, De Strooper B, Nuttin B, Debyser Z (2002) Characterization of lentiviral vector-mediated gene transfer in adult mouse brain. Hum Gene Ther 13(7):841-853

8. Lo BC, Schneider BL, Bauer M, Sajadi A, Brice A, Iwatsubo T, Aebischer P (2004) Lentiviral vector delivery of parkin prevents dopaminergic degeneration in an alpha-synuclein rat model of Parkinson's disease. Proc Natl Acad Sci USA 101(50):17510-17515

9. Lo BC, Ridet JL, Schneider BL, Deglon N, Aebischer P (2002) alpha-Synucleinopathy and selective dopaminergic neuron loss in a rat lentiviral-based model of Parkinson's disease. Proc Natl Acad Sci USA 99(16):10813-10818

10. Boska MD, Hasan KM, Kibuule D, Banerjee R, McIntyre E, Nelson JA, Hahn T, Gendelman HE, Mosley RL (2007) Quantitative diffusion tensor imaging detects dopaminergic neuronal degeneration in a murine model of Parkinson's disease. Neurobiol Dis 26(3):590-596

11. Kondoh T, Bannai M, Nishino H, Torii K (2005) 6-Hydroxydopamine-induced lesions in a rat model of hemi-Parkinson's disease monitored by magnetic resonance imaging. Exp Neurol 192(1):194-202

12. Pelled G, Bergman H, Goelman G (2002) Bilateral overactivation of the sensorimotor cortex in the unilateral rodent model of Parkinson's disease- - a functional magnetic resonance imaging study. Eur J Neurosci 15(2):389-394

13. Pelled G, Bergman H, Ben-Hur T, Goelman G (2005) Reduced basal activity and increased functional homogeneity in sensorimotor and striatum of a Parkinson's disease rat model: a functional MRI study. Eur J Neurosci 21(8):2227-2232

14. Pelled G, Bergman H, Ben-Hur T, Goelman G (2007) Manganeseenhanced MRI in a rat model of Parkinson's disease. J Magn Reson Imaging 26(4):863-870

15. Schwarting R, Huston J (1996) The unilateral 6-hydroxydopamine lesion model in behavioral brain research. Analysis of functional 
deficits, recovery and treatments. Prog Neurobiol 50(2-3):275331

16. Fischman AJ, Babich JW, Elmaleh DR, Barrow SA, Meltzer P, Hanson RN, Madras BK (1997) SPECT imaging of dopamine transporter sites in normal and MPTP-treated rhesus monkeys. J Nucl Med 38(1):144-150

17. Booij J, Andringa G, Rijks LJ, Vermeulen RJ, De Bruin K, Boer GJ, Janssen AG, Van Royen EA (1997) 123I]FP-CIT binds to the dopamine transporter as assessed by biodistribution studies in rats and SPECT studies in MPTP-lesioned monkeys. Synapse 27(3):183190

18. Scherfler C, Donnemiller E, Schocke M, Dierkes K, Decristoforo C, Oberladstatter M, Kolbitsch C, Zschiegner F, Riccabona G, Poewe W (2002) Evaluation of striatal dopamine transporter function in rats by in vivo beta-[123I]CIT pinhole SPECT. NeuroImage 17(1):128-141

19. Meissner W, Prunier C, Guilloteau D, Chalon S, Gross CE, Bezard E (2003) Time-course of nigrostriatal degeneration in a progressive MPTP-lesioned macaque model of Parkinson's disease. Mol Neurobiol 28(3):209-218

20. Perese DA, Ulman J, Viola J, Ewing SE, Bankiewicz KS (1989) A 6-hydroxydopamine-induced selective parkinsonian rat model. Brain Res 494(2):285-293

21. Thomas J, Wang J, Takubo H, Sheng J, de Jesus S, Bankiewicz KS (1994) A 6-hydroxydopamine-induced selective Parkinsonian rat model: further biochemical and behavioral characterization. Exp Neurol 126(2):159-167

22. Perese DA, Ulman J, Viola J, Ewing SE, Bankiewicz KS (1989) A 6-hydroxydopamine-induced selective parkinsonian rat model. Brain Res 494(2):285-293

23. Ungerstedt U (1971) Striatal dopamine release after amphetamine or nerve degeneration revealed by rotational behaviour. Acta Physiol Scand 367:49-68

24. Votaw J, Byas-Smith M, Hua J, Voll R, Martarello L, Levey AI, Bowman FD, Goodman M (2003) Interaction of isoflurane with the dopamine transporter. Anesthesiology 98(2):404-411

25. Votaw JR, Byas-Smith MG, Voll R, Halkar R, Goodman MM (2004) Isoflurane alters the amount of dopamine transporter expressed on the plasma membrane in humans. Anesthesiology 101(5):1128-1135

26. Bequé D, Vanhove C, Andreyev A, Nuyts J, Defrise M (2004) Correction for imperfect camera motion and resolution recovery in pinhole SPECT. Conference proceedings of IEEE medical imaging conference, Rome, Italy M2-173

27. Bequé D, Nuyts J, Suetens P, Bormans P (2005) Optimization of geometrical calibration in pinhole SPECT. IEEE Trans Med Imaging 24(5):667-675

28. Casteels C, Vermaelen P, Nuyts J, Van Der LA, Baekelandt V, Mortelmans L, Bormans G, Van LK (2006) Construction and evaluation of multitracer small-animal PET probabilistic atlases for voxel-based functional mapping of the rat brain. J Nucl Med 47(11):1858-1866

29. Hall S, Rutledge JN, Schallert T (1992) MRI, brain iron and experimental Parkinson's disease. J Neurol Sci 113(2):198-1208

30. Kondoh T, Bannai M, Nishino H, Torii K (2005) 6-Hydroxydopamine-induced lesions in a rat model of hemi-Parkinson's disease monitored by magnetic resonance imaging. Exp Neurol 192(1):194-202

31. Casteels C, Lauwers E, Bormans G, Baekelandt V, Van LK (2008) Metabolic-dopaminergic mapping of the 6-hydroxydopamine rat model for Parkinson's disease. Eur J Nucl Med Mol Imaging 35(1):124-134
32. Araujo DM, Cherry SR, Tatsukawa KJ, Toyokuni T, Kornblum HI (2000) Deficits in striatal dopamine D(2) receptors and energy metabolism detected by in vivo microPET imaging in a rat model of Huntington's disease. Exp Neurol 166(2):287-297

33. Booij J, de Bruin K, de Win MML, Lavini C, den Heeten GJ, Habraken JBA (2003) Imaging of striatal dopamine transporters in rat brain with single pinhole SPECT and co-aligned MRI is highly reproducible. Nucl Med Biol 30(6):643-649

34. Baete K, Nuyts J, Laere KV, Van Paesschen W, Ceyssens S, De Ceuninck L, Gheysens O, Kelles A, Vanden Eynden J, Suetens P, Dupont P (2004) Evaluation of anatomy based reconstruction for partial volume correction in brain FDG-PET. NeuroImage 23(1):305-317

35. Kosta P, Argyropoulou MI, Markoula S, Konitsiotis S (2006) MRI evaluation of the basal ganglia size and iron content in patients with Parkinson's disease. J Neurol 253(1):26-32

36. Vymazal J, Righini A, Brooks R, Canesi M, Mariani C, Leonardi M, Pezzoli G (1999) T1 and T2 in the brain of healthy subjects, patients with Parkinson disease, and patients with multiple system atrophy: relation to iron content. Radiology 211(2):489-495

37. Ye F, Allen P, Martin W (1996) Basal ganglia iron content in Parkinson's disease measured with magnetic resonance. Mov Disord 11(3):243-249

38. Brex PA, Parker GJ, Leary SM, Molyneux PD, Barker GJ, Davie CA, Thompson AJ, Miller DH (2000) Lesion heterogeneity in multiple sclerosis: a study of the relations between appearances on T1 weighted images, T1 relaxation times, and metabolite concentrations. J Neurol Neurosurg Psychiatry 68(5):627-632

39. Paxinos G (1995) The rat nervous system. 2 edn. Academic Press, California

40. Hsu JL, Jung TP, Hsu CY, Hsu WC, Chen YK, Duann JR, Wang HC, Makeig S (2007) Regional CBF changes in Parkinson's disease: a correlation with motor dysfunction. Eur J Nucl Med Mol Imaging 34(9):1458-1466

41. Yang J, Sadler TR, Givrad TK, Maarek JM, Holschneider DP (2007) Changes in brain functional activation during resting and locomotor states after unilateral nigrostriatal damage in rats. NeuroImage 36(3):755-773

42. Bezard E, Gross CE, Brotchie JM (2003) Presymptomatic compensation in Parkinson's disease is not dopamine-mediated. Trends Neurosci 26(4):215-221

43. Dahlgren N, Lindvall O, Nobin A, Stenevi U (1981) Cerebral circulatory response to hypercapnia: effects of lesions of central dopaminergic and serotoninergic neuron systems. Brain Res 230(1-2): 221-233

44. Lindvall O, Ingvar M, Stenevi U (1981) Effects of methamphetamine on blood flow in the caudate-putamen after lesions of the nigrostriatal dopaminergic bundle in the rat. Brain Res 211(1):211216

45. Mraovitch S, Calando Y, Onteniente B, Peschanski M, Seylaz J (1993) Cerebrovascular and metabolic uncoupling in the caudateputamen following unilateral lesion of the mesencephalic dopaminergic neurons in the rat. Neurosci Lett 157(2):140-144

46. Choi JK, Chen YI, Hamel E, Jenkins BG (2006) Brain hemodynamic changes mediated by dopamine receptors: Role of the cerebral microvasculature in dopamine-mediated neurovascular coupling. NeuroImage 30(3):700-712 